\title{
Emergence and transmission of misinformation in the context of social interactions
}

\author{
Magda Saraiva $^{1}$ (D) - Margarida Vaz Garrido ${ }^{1} \cdot$ Pedro B. Albuquerque $^{2}$ \\ Published online: 12 August 2020 \\ (C) The Psychonomic Society, Inc. 2020
}

\begin{abstract}
The emergence and transmission of false memories is well documented in individual memory tasks. However, the examination of these processes in the context of social interaction still presents mixed findings. The present study further examines the potential of collaboration in minimizing the acceptance and retrieval of misinformation. In Experiment 1 participants watched a video immediately followed by a recall task (collaborative vs. nominal). Then a questionnaire (collaborative vs. individual) containing true information and misinformation about the video was presented. After the questionnaire, participants were given a new recall task (collaborative vs. nominal). We expected that collaboration at encoding and at retrieval would reduce the acceptance and recall of misinformation. Results revealed, as expected, that collaborative groups performed better in answering the questionnaire, accepting more correct information and rejecting more misinformation. Subsequently, they also recalled less misinformation. However, their recall of correct information was also lower. To rule out the potential role of collaborative inhibition in explaining the results observed in the final recall, in Experiment 2 the collaborative manipulation occurred only during the questionnaire and both recall tasks were individual. Again, participants answering the questionnaire collaboratively performed better than those answering individually. Critically, in a subsequent individual recall task, they produced less false memories and more correct information than those answering the questionnaire individually. These results suggest that collaboration during information encoding reduces the acceptance of misinformation and its subsequent recall.
\end{abstract}

Keywords Misinformation $\cdot$ Social interaction $\cdot$ False memories $\cdot$ Collaborative inhibition

\section{Introduction}

The production of false memories in individual memory tasks has been extensively studied with several paradigms (see Oliveira, Albuquerque, \& Saraiva, 2018, for a review). One of the most well established paradigms to investigate false memories - DRM (Deese, 1959; Roediger \& McDermott, 1995) consists of presenting a list of words (e.g., legs, seat, soft, desk, arm, wood, cushion, rest) that are associated with another word, the critical lure (e.g., chair), which is never presented. The main finding observed with this paradigm consistently revealed that individuals frequently recognize/recall

Magda Saraiva

magda.saraiva@iscte-iul.pt

1 Centro de Investigação e Intervenção Social, Iscte-Instituto Universitário de Lisboa, Avenida das Forças Armadas, 1649-026 Lisboa, Portugal

2 Escola de Psicologia, Departamento de Psicologia Básica, Universidade do Minho, Braga, Portugal the critical lure as having been presented in the initial word list (see Gallo, 2006, for a review). Another experimental procedure often used to study false memories is the misinformation paradigm (Loftus \& Palmer, 1974). This paradigm rests on the distortion of memory for information/events by introducing false or misleading information after initial encoding. In this paradigm, participants are initially presented with an image or a video. False or misleading information about the information initially presented is then introduced through a questionnaire or a narrative. Finally, in the retrieval phase, participants are asked to remember as much information as possible from what was initially presented through a recall or a recognition task. The main findings observed with this paradigm have shown that people tend to recall/recognize the false or misleading information as being true (Loftus, 1975).

One of the most common explanations for the emergence of false memories in the two paradigms is the sourcemonitoring framework (Johnson, Hashtroudi, \& Lindsay, 1993; Roediger, Meade, \& Bergman, 2001). According to this account, the memory errors observed derive from individual failure in monitoring the source of information (critical lure or 
misinformation). In other words, participants attribute the source of the critical lure to the original word list (in the DRM) and the misinformation to the original event (in the misinformation paradigm) and not to the false information source (e.g., critical lure activation processes, or questionnaire, narrative, confederate, respectively) (e.g., Johnson et al., 1993; Meade \& Roediger, 2002; Roediger et al., 2001).

Despite both paradigms leading to the production of false information, the nature of that information is different (e.g., Loftus, 2005; Otgaar \& Candel, 2011). In the DRM, the production of false information is spontaneous, insofar as it is generated by the individual through internal processes of semantic activation of the critical lure, without external suggestion (Otgaar \& Candel, 2011). The false information produced in the misinformation paradigm is based on the suggestion inserted through post-event misinformation that is misattributed to the memory for the original event (e.g., Loftus, 2005; Otgaar, Candel, Scoboria, \& Merckelbach, 2010; Zhu, Chen, Loftus, Lin, \& Dong, 2013). Additionally, the production of false memories in the two paradigms seems to be weakly related to each other (e.g., Ost et al., 2013; Otgaar \& Candel, 2011). For example, while Ost et al. (2013) report robust DRM and misinformation effects within the same participants, no correlations were observed between misinformation and DRM measures, suggesting that the two types of false memories are not "equivalent." A similar pattern was previously reported by Otgaar and Candel (2011, Exp. 1), where no relation between the susceptibility to the DRM effect and acquiescing to suggestive information was observed in the same group of children.

The production of false memories is well established in individual memory tasks. However, memory often occurs as a cooperative and social activity (Garcia-Marques, Garrido, Hamilton, \& Ferreira, 2012; Garrido, Garcia-Marques, \& Hamilton, 2012a, b; Rajaram \& Pereira-Pasarin, 2010). Notably, while the emergence and transmission of false information are well established in individual memory tasks, their examination in the context of social interaction still presents mixed findings.

The production of false memories in groups has typically been examined in collaborative memory tasks, that is, in groups of two or more individuals remembering together information that was experienced together or individually (e.g., Meade, Nokes, \& Morrow, 2009). These studies have revealed that collaborative recall is higher than individual recall (Maki, Weigold, \& Arellano, 2008; Weldon \& Bellinger, 1997). However, the critical comparison between collaborative and individual recall requires the use of nominal groups; that is, the sum of non-redundant individually recalled items of as many group members as those that constitute the collaborative group (Rajaram \& Pereira-Pasarin, 2010). This comparison has consistently shown a counterintuitive result: nominal groups recall more information than collaborative ones (e.g., Harris, Barnier, \& Sutton, 2012; Weldon \& Bellinger, 1997; see Rajaram \& Pereira-Pasarin, 2010, for a review). This phenomenon is known as the collaborative inhibition effect (Weldon \& Bellinger, 1997). Among the various attempts to explain this effect, the disruption of retrieval strategies hypothesis proposed by Basden, Basden, Bryner, and Thomas (1997) has been the most documented. According to this hypothesis, each group member develops idiosyncratic strategies for organizing information during the encoding phase. During collaborative retrieval, the recall outputs from each group member may disrupt the organization of the recall strategy encoded by each one of them, resulting in poor group performance (Basden et al., 1997).

In addition to the collaborative inhibition effect, some authors argue that another cost of collaborative memory is the higher production of false memories compared to nominal groups (Thorley \& Dewhurst, 2007). However, while some studies using the DRM found that the production of false memories in collaborative groups is higher than in nominal groups (Thorley \& Dewhurst, 2007), other studies reported the opposite result (Saraiva, Albuquerque, \& Arantes, 2017). Still other studies did not find significant differences between collaborative and nominal groups in the production of false memories (Basden, Basden, Thomas, \& Souphasith, 1998).

Further attempts to study false memories in the context of social interaction using the misinformation paradigm (Loftus \& Palmer, 1974) have also reported contradictory results. For example, in the study by KarnsWright, Irvin, Suranic, and Rivardo (2009), participants watched a video of a car accident and then read a narrative introducing correct information or misinformation. Finally, they answered to a set of questions about the video, collaboratively or individually. The results revealed that the misinformation effect was smaller in collaborative groups (vs. individual). However, Rivardo et al. (2013) reached a somewhat different conclusion. In a similar procedure to that used by Karns-Wright et al. (2009), after responding collaboratively or individually to the questionnaire (time 1), participants individually answered the questionnaire again, immediately (time 2) and 1 week later (time 3). As expected, participants who were exposed to the narrative containing inaccurate information were more likely to report misinformation than those who received the accurate narrative (at both time 1 and time 2). Critically, 1 week later (time 3), participants who collaborated at time 1 reported more misinformation than those who answered individually (Rivardo et al., 2013). Recently, Rossi-Arnaud et al. (2020) reported similar results to those obtained by Karns-Wright et al. (2009). After watching a video, participants performed an individual or collaborative recall task. Then they answered (collaboratively vs. individually) to a set of questions, some of which contained 
misinformation. After a delay of 1 hour or 1 week, the results from a recognition task (collaborative vs. individual) indicated that collaborative groups were less likely to provide false assents to misleading statements, regardless of the delay.

Finally, the influence of social sources on memory has also been examined as "social contagion" (e.g., Meade \& Roediger, 2002; Roediger et al., 2001) by combining the assumptions of misinformation and conformity (Gabbert, Memon, \& Allan, 2003) paradigms. In these studies, after the presentation of information (e.g., household scenes) to a single participant (e.g., Meade \& Roediger, 2002) or a group of naïve participants (e.g., McNabb \& Meade, 2014), misinformation is introduced by a social source (e.g., confederate) during a collaborative recall task. The results have shown that in a final individual recall task, naïve participants retrieve the false information previously recalled by the confederate.

The mixed results reported in the examination of false memories in collaborative memory tasks, regardless of the paradigm used, are likely to stem from procedural and methodological differences (e.g., method of recall; associative strength of word lists to critical lure; group size; among others). Among all these variables, the collaboration method used in these tasks seems to play an important role in shaping the emergence of false memories. For example, in their comparison between the two collaboration methods typically used in collaborative memory tasks (i.e., free-for-all and turn-taking) using the DRM paradigm, Thorley and Dewhurst (2007) observed more false memories in groups collaborating with the turn-taking method. More recently, Harris et al. (2012) compared the costs and benefits of turn-taking and reaching consensus collaboration procedures. The results of a final individual recall task showed that participants in the consensus group were more accurate than those in the turn-taking and nominal groups. This was arguably the case, because in the consensus condition participants engaged in group source-monitoring processes, unlike the group in the turn-taking condition. These results suggest the relevance of group discussion for memory accuracy and were further supported by other studies showing that under certain conditions discussion can improve memory. For example, Vredeveldt, Hildebrandt, and Van Koppen (2016), and Vredeveldt, Groen, Ampt, and van Koppen (2017) reported that pairs of participants who discussed the information to be remembered produced fewer errors and recalled more new information in a subsequent individual recall task than nominal pairs.

Overall, these findings converge in that different collaboration methods significantly influence the information produced (see Marion \& Thorley, 2016, and Maswood \& Rajaram, 2019, for reviews). While the free-for-all method and reaching consensus allow and encourage interaction between group members and consequent discussion of ideas, in the turn-taking method the interaction between group members is limited, increasing the pressure to recall and consequently the production of errors (Harris et al., 2012; Maswood \& Rajaram, 2019; Vredeveldt et al., 2016, 2017).

According to the retrieval criterion shift account (e.g., Takahashi, 2007; Thorley \& Dewhurst, 2007), while the free-for-all method prompts the group to adopt a more conservative retrieval criterion because the social risks of making errors is higher, the turn-taking method is likely to lead group members to use a less conservative retrieval criterion, which increases the production of false memories (Basden et al., 1997; Thorley \& Dewhurst, 2007; see Maswood \& Rajaram, 2019, for a review).

Additionally, the potential benefit of collaboration in minimizing the production of false memories also lies in errorpruning processes (e.g., Rajaram \& Pereira-Pasarin, 2010; Ross, Spencer, Blatz, \& Restorick, 2008). According to this explanation, retrieval collaboration using the free-for-all method or reaching consensus (vs. the turn-taking method) offers an opportunity to discuss ideas and receive corrective feedback, which reduces the production of errors (e.g., Weigold, Russell, \& Natera, 2014).

Taken together, these arguments seem to indicate that collaboration methods such as free-for-all or reaching consensus increase the rejection of misinformation by comparison with the turn-taking method, either by promoting the adoption of a more conservative retrieval criterion or by correction processes arising from the discussion.

\section{The present study}

The present study further investigates the emergence of misinformation in social interaction contexts, examining whether collaborating with the free-for-all method at encoding and retrieval might reduce misinformation acceptance.

To this end, after having participants watching a video, misinformation was introduced by a questionnaire, including false or misleading statements. Unlike previous studies using the misinformation paradigm in collaborative contexts, we included a recall task (R1) immediately after the presentation of a video to obtain a baseline of participants' memory capacity prior to the introduction of misinformation. This baseline permitted the direct comparison of recall performance before and after misinformation was presented. Additionally, during the questionnaire, participants working in collaboration were offered the opportunity to immediately discuss the information presented, thus providing an indicator of whether they more effectively reject misinformation when working in collaboration than individually. Finally, instead of a cued recall or a recognition task, typically used in previous studies, we 
used a free-recall task after misinformation was presented (R2), as this task is more likely to directly reflect the integration of misinformation in the group's memory.

We expected to observe the benefits of collaboration both during the encoding of misinformation and in a subsequent recall task. Because participants were allowed to freely discuss information during the tasks, those in the collaborative condition were expected to provide more correct responses to the questionnaire and to accept more correct information and less misinformation than those who answered the questionnaire individually. Participants in collaborative conditions were also expected to introduce more correct information and less misinformation from the questionnaire in R2.

\section{Experiment 1}

Experiment 1 aimed to further explore the acceptance and recall of misinformation in collaborative groups. After watching a video, the encoding and recall of misinformation of nominal and collaborative groups was compared. The first recall (R1) occurred immediately after the video presentation, while the second recall (R2) occurred after the introduction of a questionnaire containing true information and misinformation.

We expected that participants responding in collaboration to the questionnaire would correctly respond to more statements than those responding individually because they were allowed to freely discuss information during the task. Critically, the potential benefits of collaboration in minimizing the acceptance of misinformation should also be observed in the final recall task.

\section{Method}

Participants A sample of 76 pairs of participants was determined by an a priori power analysis ( $\mathrm{G}^{*}$ Power), using as reference a medium to large effect size $\left(\eta_{p}{ }^{2}=.10\right)$ and a power $1-\beta=0.80$ to detect the interaction between Group (Nominal vs. Collaborative) and Accepted Information (Misinformation vs. Correct; within participants). A total of 152 participants volunteered for the experiment $\left(106 \mathrm{~F} ; M_{\text {age }}=21.74 ; S D=\right.$ 5.32). Participants were randomly divided into 38 collaborative pairs and 38 nominal pairs.

Design The performance in the questionnaire was analyzed in a 2 (Condition: Individual vs. Collaborative; between-participants) $\times 2$ (Type of accepted information: Misinformation vs. Correct; within-participants) mixed design. The recall of misinformation and correct information introduced by the questionnaire was examined in a 2 (Group: Nominal vs. Collaborative; between-participants) $\times 2$ (Type of recalled information: Misinformation vs. Correct; within-participants) mixed design.

Materials We selected a 3-min video about a bank robbery without signs of violence (Herrington, 2002). The misinformation about the video was introduced in a questionnaire adapted from Luna and Migueles (2008, 2009). The questionnaire consisted of 32 true or false questions, of which 16 contained correct information and the remaining 16 containing false or misleading information about the video. False information corresponded to information that was not presented in the video (e.g., "the bank secretary had a scarf tied around her neck" - when she did not), while misleading information corresponded to distorted information about the video (e.g., "the guards got out of the security car through the back door" - when they actually came out from the side door).

\section{Procedure}

Participants arrived at the laboratory to collaborate in a study about information processing in groups. No reference was ever made to the production of false memories. All procedures were conducted in line with the ethical guidelines of the host institution, and written consent was obtained.

The pairs of participants sat in separate tables, each facing a 17 -in. monitor where the video was presented. Participants were instructed to pay close attention to the video. After watching the video, they performed a distracting word search task for $2 \mathrm{~min}$. Then they were asked to remember (R1) and write down as many details of the video as possible such as actions, people, objects or colors. Participants in nominal groups performed this task individually. Participants in collaborative groups performed the task using the free-for-all method. Specifically, they were instructed to freely discuss everything they remembered, to resolve any disagreements that may arise, and to reach consensus about the information they would write down. After the recall task, participants were given the questionnaire. In the individual condition, participants answered the questionnaire alone and in silence. Participants in the collaborative condition were instructed to discuss the answers to each question and to give a single answer for the group. Once again, they should reach a consensus in case of disagreement.

After a 2-min distracting task, participants were asked to perform a second collaborative (i.e., collaborative groups) or individual (i.e., nominal groups) recall task (R2). The outputs of R2 constituted an indicator of the amount of misinformation that participants integrated in their memory. The total duration of the experiment was about $40 \mathrm{~min}$. At the end of the experiment, participants were thanked and debriefed. 


\section{Data analysis ${ }^{1}$}

A comprehensive list of video details, including actions, objects, people, colors, etc., was compiled in 132 information units (e.g., car, blue, tie, bags; Paulo, Albuquerque, Saraiva, \& Bull, 2015). Recalled information was classified as either correct (i.e., correct details recalled) or distortion (i.e., self-generated incorrect information). In both recall tasks, the correct units of recalled information from the video were identified and coded as 1 and each distortion as 0 . The coding of the recalled information was made by two independent raters, one of them completely naïve to the goals of the study, and disagreements between raters were resolved by a third independent rater. Inter-rater agreement was very high for all dependent variables (Recall 1: Individual recall $-\kappa=.882,95 \% \mathrm{CI}[.83, .94], p<.001$; Collaborative recall $-\kappa=$ $.854,95 \%$ CI $[.79, .92], p<.001$; Recall 2: Individual recall $-\kappa=$ $.874,95 \%$ CI [.82,.93], $p<.001$; Collaborative recall $-\kappa=.863$, $95 \%$ CI $[.80, .92], p<.001)$.

In order to compare collaborative and individual performance in both recall tasks, nominal groups were created (Weldon \& Bellinger, 1997; see Marion \& Thorley, 2016, for a review). The correctly recalled information was calculated by adding all information units coded with 1 and the distortions by counting coded with all 0 . For nominal groups, all the redundant information items (correct or distortions) were eliminated.

The proportion of correct recall was calculated by dividing the number of correct units of information recalled by the 132 possible. To analyze the recall of the information introduced by the questionnaire, we identified the number of correct (16 maximum) and misinformation (16 maximum) information units recalled in $\mathrm{R} 2$ and calculated the respective proportions. ${ }^{2}$

\section{Results $^{3}$}

\section{Recall 1}

Collaborative groups recalled an average of $37.29(S D=$ 12.03) information units, of which $7.76(S D=3.49)$ were

\footnotetext{
${ }^{1}$ Prior to all analyses reported, relevant assumptions were checked. For some variables the homogeneity of the variance was not guaranteed. In these cases, the non-parametric tests conducted confirmed the results of the parametric tests. Additionally, some analyses of interest, namely interaction effects, are not possible with non-parametric tests. For these reasons and to allow comparisons with other dependent variables, we reported the results of the parametric tests.

${ }^{2}$ If a correct or a false information item had already been recalled in R1, in calculating the proportion of accepted information from the questionnaire in R2, that information was discounted. For example, if one item of correct information presented in the questionnaire was already recalled in the first recall task, the proportion was $\mathrm{X} / 15$ instead of $\mathrm{X} / 16$.

${ }^{3}$ The raw data and the syntax used in the reported analyses are available at O S F ( h t p s : / / o s f. i o / d n g ph/? vi e w o n ly = 963296c71b16448f8ca39e63209ac275).
}

distortions, while nominal groups recalled an average of $54.66(S D=13.41)$ information units, of which $7.79(S D=$ 4.17) were distortions.

The recall proportion of correct information in collaborative groups was significantly lower $(M=.22 ; S D=.08)$ than that of nominal groups $(M=.36 ; S D=.09)$, replicating the collaborative inhibition effect, $t(74)=6.99, p<.001, d=1.60$, $90 \%$ CI $[1.16,2.03]$. The difference in the number of distortions observed between collaborative and nominal groups was not significant, $t(74)=.030, p=.976$.

\section{Correct information and misinformation accepted during the questionnaire}

Participants' correct responses to the questionnaire were calculated by adding the number of "true" responses to the questions that matched information presented in the video, and the number of "false" responses to the questions containing information that did not match the information presented. Participants who responded collaboratively to the questionnaire provided significantly more correct responses $(M=$ 23.63, $S D=2.12$ ) than those who responded individually ( $M$ $=21.78, S D=2.62), t(112)=-3.79, p<.001, d=-.75,90 \% \mathrm{CI}$ $[.42,1.09]$.

Additionally, we compared the acceptance of correct information (i.e., to consider that a statement is true when it corresponds to information presented in the video) and misinformation $^{4}$ (i.e., to consider that a statement is true when the information is new and does not correspond to information presented in the video) between participants responding to the questionnaire collaboratively or individually. A mixed ANOVA 2 (Condition: Individual vs. Collaborative) $\times 2$ (Type of accepted information: Misinformation vs. Correct) revealed a significant main effect of the type of accepted information, $F(1,112)=750.06, p<.001, \eta p^{2}=.870,90 \% \mathrm{CI}$ $[.83, .89]$, indicating that correct information $(M=12.89, S D=$ 1.73) was more accepted than misinformation $(M=6.50, S D=$ 2.13). The main effect of condition was not significant, $F(1,112)=.232, p=.63$, that is, the groups did not differ significantly in the overall amount of information accepted from the questionnaire. Importantly, the interaction between type of information and condition was significant, $F(1,112)=$ $14.36, p<.001, \eta p^{2}=.114,90 \%$ CI $[.04, .21]$ (see Table 1 ). Planned comparison showed that participants in both individual, $F(1,112)=417.64, p<.001, \eta p^{2}=.789,90 \%$ CI $[.73$, .83 ], and collaborative conditions, $F(1,112)=364.50, p<$ $.001, \eta p^{2}=.765,90 \%$ CI $[.70, .81]$, accepted more correct information than misinformation. Importantly, participants

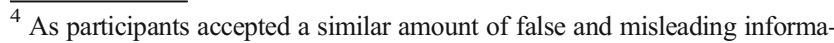
tion in both conditions - Collaborative, $t(37)=-.251, p=.80$; Control condition (individual), $t(75)=.416, p=.68-$ we collapsed these two types of information into a single "misinformation" index.
} 
Table 1 Correct information and misinformation accepted during the questionnaire by condition

\begin{tabular}{lll}
\hline Condition & $\begin{array}{l}\text { Correct information accepted } \\
M(S D)\end{array}$ & $\begin{array}{l}\text { Misinformation accepted } \\
M(S D)\end{array}$ \\
\hline Individual & $12.54(1.81)$ & $6.76(2.22)$ \\
Collaborative & $13.61(1.31)$ & $5.97(1.85)$ \\
Total & $12.89(1.73)$ & $6.50(2.13)$ \\
\hline
\end{tabular}

who answered the questionnaire in collaboration accepted more correct information, $F(1,112)=10.40, p=.002, \eta p^{2}=$ $.085,90 \% \mathrm{CI}[.02, .17]$, than those who answered the questionnaire individually. In contrast, collaborative groups accepted less misinformation than individuals working alone, but this difference did not reach statistical significance, $F(1,112)=3.56 p=.06, \eta p^{2}=.031,90 \%$ CI $[.00, .10]$.

\section{Recall 2}

In R2, collaborative groups recalled an average of 37.00 information units $(S D=12.85)$, of which $7.26(S D=4.22)$ were distortions, whereas nominal groups recalled an average of $55.03(S D=12.23)$ information units, of which $7.82(S D=$ 3.94) were distortions.

The amount of correct information (from the original event) retrieved during the R2 was significantly lower in collaborative $(M=.22 ; S D=.08)$ than in nominal groups $(M=$ $.36 ; S D=.08)$, replicating the collaborative inhibition effect, $t(74)=7.41, p<.001, d=1.70,90 \%$ CI $[1.25,2.14]$. The groups did not differ significantly in terms of distortions, $t(74)$ $=.590, p=.56$.

To analyze whether the groups' recall of correct information from the original event varied from the first to the second recall task, we conducted a 2 (Recall task: R1 vs. R2; withinparticipants) $\times 2$ (Group: Nominal vs. Collaborative; betweenparticipants) mixed ANOVA. The observed main effect of group, $F(1,74)=53.16, p<.001, \eta p^{2}=.418,90 \%$ CI $[.27$, .53], replicates the collaborative inhibition effect across both recall tasks $\left(M_{\mathrm{Nom}}=.36, S D=.09 ; M_{\mathrm{Coll}}=.22, S D=.08\right)$. The main effect of recall task and the interaction effect between recall task and group were not significant, $F(1,74)=.033, p=$ .86 , and $F(1,74)=.606, p=.44$, respectively.

\section{Recall of the information presented in the questionnaire $^{5}$}

To investigate whether the benefits of collaboration observed in the questionnaire would still be detected in a subsequent recall task we conducted a 2 (Group: Nominal vs. Collaborative;

\footnotetext{
${ }^{5}$ Correct information and misinformation introduced by the questionnaire recalled in $\mathrm{R} 2$.
}

between subject) $\times 2$ (Type of recalled information: Correct vs. Misinformation) mixed ANOVA. A significant main effect of group, $F(1,74)=11.61, p=.001, \eta p^{2}=.136,90 \%$ CI $[.04$, $.24]$, indicated that nominal groups $(M=.24, S D=.18)$ recalled significantly more information introduced by the questionnaire than collaborative groups $(M=.16, S D=.16)$. The main effect of type of information was also significant, $F(1,74)=187.78, p$ $<.001, \eta p^{2}=.717,90 \%$ CI $[.62, .77]$, revealing that participants recalled more correct information $(M=.32, S D=.16)$ than misinformation $(M=.08, S D=.08)$ from the questionnaire. The interaction between group and type of recalled information was also significant, $F(1,74)=4.74, p=.03, \eta p^{2}=.060,90 \%$ CI $[.00, .16]$ (see Fig. 1).

Planned comparisons showed that both nominal, $F(1,74)=$ $126.09, p<.001, \eta p^{2}=.630,90 \%$ CI $[.51, .70]$, and collaborative groups, $F(1,74)=66.43, p<.001, \eta p^{2}=.473,90 \% \mathrm{CI}$ $[.33, .57]$ recalled more correct $\left(M_{\mathrm{Nom}}=.37, S D=.15 ; M_{\text {Coll }}=\right.$ $.26, S D=.16)$ than misinformation $\left(M_{\mathrm{Nom}}=.10, S D=.09\right.$; $M_{\text {Coll }}=.06, S D=.07$ ) introduced by the questionnaire. As expected, collaborative groups recalled less misinformation, $F(1,74)=4.73, p=.03, \eta p^{2}=.060,90 \%$ CI $[.00, .16]$ than nominal groups. However, they also recalled less correct information, $F(1,74)=10.45, p=.002, \eta p^{2}=.124,90 \% \mathrm{CI}[.03$, $.24]$, than nominal groups.

Experiment 1 showed that participants who answered the questionnaire collaboratively provided more correct responses, accepted more correct information, and tended to reject more misinformation than those who worked individually. However, this advantage was not directly observed in a subsequent recall task where, while recalling less misinformation, collaborative groups also recalled less correct information. This might have been the case due to a collaborative inhibition effect that generalized across both correct and misinformation.

\section{Experiment 2}

To uncover the influence of collaborative inhibition in the results observed in the previous experiment, in Experiment 2 , the collaborative manipulation was only introduced during the questionnaire (i.e., collaborative vs. individual), and the recall tasks were always individual. We expected that participants collaborating during the questionnaire would accept more correct information and reject more misinformation, and that this advantage would still be observed during a subsequent individual recall task.

\section{Method}

Participants A sample of 76 pairs of participants was determined as in Experiment 1. A total of 152 participants 


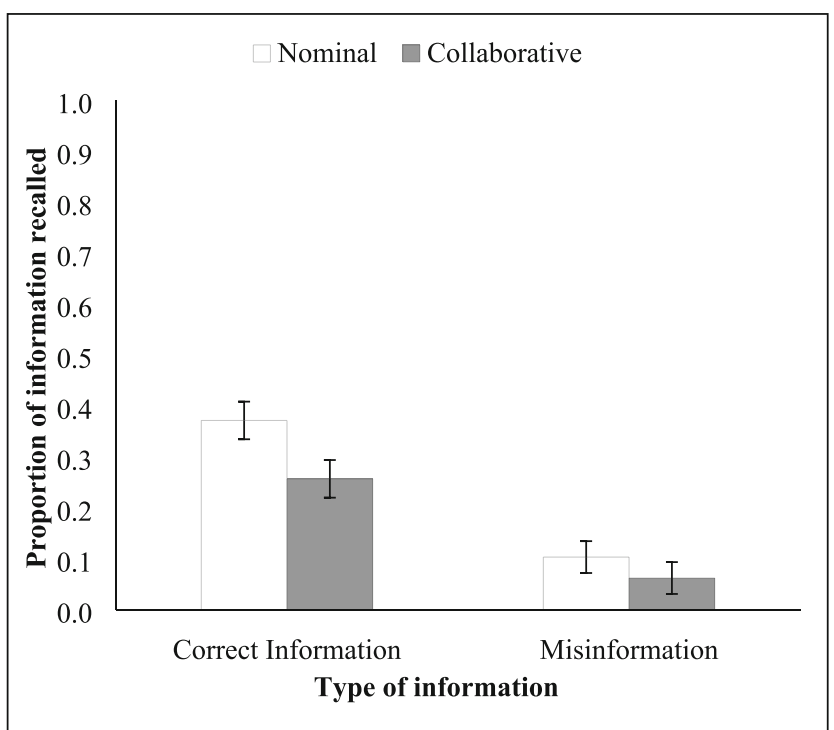

Fig. 1 Proportion of correct information and misinformation recalled from the questionnaire in Recall 2 by condition (error bars indicate $95 \%$ confidence interval)

volunteered for the experiment $\left(119 \mathrm{~F} ; M_{\text {age }}=21.93 ; S D=\right.$ 6.17). Half of them answered the questionnaire in groups of 2 $(N=38$ pairs $)$ and the remaining individually (control condition $^{6}$ ).

Design The role of collaboration in the encoding of misinformation and correct information was examined in a 2 (Condition: Individual vs. Collaborative; between-participants) $\times 2$ (Type of Accepted information: Misinformation vs. Correct; within-participants) mixed design. Performance during the questionnaire was analyzed in a similar way as in Experiment 1. Finally, recall was examined in a 2 (Recall: R1 vs. R2; within participants) $\times 2$ (Condition: Individual vs. Collaborative $^{7}$; between-participants) mixed design.

Materials and procedure The same as in Experiment 1, with the exception that both recall tasks were individual.

\section{Results}

Inter-rater agreement was very high for all dependent variables (Recall 1: Collaborative group $-\kappa=.842,95 \%$ CI $[.78, .91], p<.001$; Recall 2: Collaborative group $-\kappa=$ $.803,95 \%$ CI $[.73, .87], p<.001)$; see inter-rater agreement for individual recall reported in Experiment 1.

\footnotetext{
${ }^{6}$ Participants in the control condition were the same as those who participated in Experiment 1, since the procedure for this condition was exactly the same. ${ }^{7}$ In this experiment, the collaborative manipulation was only introduced during the questionnaire. Yet we kept the label "condition" in the entire design to distinguish the two groups.
}

\section{Recall 1}

The first recall was individual for both "conditions," thus a similar recall performance was expected. Participants recalled an average of $34.25(S D=8.75)$ and $34.79(S D=11.19)$ information units, of which $4.42(S D=2.00)$ and $3.89(S D=$ 2.32) were distortions, respectively. No significant differences in the proportion of correct recall $(M=.23 ; S D=.06$ and $M=$ $.23 ; S D=.08), t(150)=.697, p=.49$ or in distortions between the two conditions, $t(150)=-1.50, p=.14$, were observed.

\section{Correct information and misinformation accepted during the questionnaire}

Overall, the results replicated those observed in Experiment 1. Participants who responded collaboratively to the questionnaire $(M=23.76, S D=2.38)$ provided significantly more correct responses than those who responded individually ( $M$ $=21.78, S D=2.62), t(112)=-3.94, p<.001, d=-782,90 \%$ CI $[.45,1.12]$.

A mixed ANOVA 2 (Condition: Individual vs. Collaborative) $\times 2$ (Type of accepted information: Misinformation vs. Correct) showed a significant main effect of the type of accepted information, $F(1,112)=$ $720.34, p<.001, \eta p^{2}=.865,90 \%$ CI $[.83, .89]$, indicating that correct information $(M=12.93, S D=1.78)$ was more accepted than misinformation ${ }^{8}(M=6.49, S D=$ 2.19). The main effect of the condition was not significant, $F(1,112)=.367, p=.55$, indicating that the overall amount of information accepted from the questionnaire did not differ significantly between conditions. Finally, the interaction between type of information and condition was significant, $F(1,112)=15.51, p<.001, \eta p^{2}=.122$, $90 \%$ CI $[.04, .22]$. Planned comparisons revealed that participants in both individual, $F(1,112)=393.33, p<$ $.001, \eta p^{2}=.778,90 \%$ CI $[.72, .82]$, and collaborative conditions, $F(1,112)=355.22, p<.001, \eta p^{2}=.760$, $90 \%$ CI $[.70, .80]$, accepted more correct information than misinformation (see Table 2). Critically, participants who answered the questionnaire in collaboration accepted more correct information, $F(1,112)=12.06, p=.001, \eta p^{2}$ $=.097,90 \% \mathrm{CI}[.03, .19]$, than those who answered the questionnaire individually. In contrast, collaborative groups accepted less misinformation than individuals working alone, but this difference did not reach statistical significance, $F(1,112)=3.59, p=.061, \eta p^{2}=.031$, $90 \%$ CI $[.00, .10]$.

\footnotetext{
${ }^{8}$ As in Experiment 1, there were no significant differences in the acceptance of false and misleading information between the two conditions, $t(37)=-.095, p$ $=.93$. For this reason, we collapsed these two types of information into a single "misinformation" index.
} 
Table 2 Correct information and misinformation accepted during questionnaire by condition

\begin{tabular}{lll}
\hline Condition & $\begin{array}{l}\text { Correct information accepted } \\
M(S D)\end{array}$ & $\begin{array}{l}\text { Misinformation accepted } \\
M(S D)\end{array}$ \\
\hline Individual & $12.54(1.81)$ & $6.76(2.22)$ \\
Collaborative & $13.71(1.43)$ & $5.95(2.05)$ \\
Total & $12.93(1.78)$ & $6.49(2.19)$ \\
\hline
\end{tabular}

\section{Recall 2}

In R2, participants who answered the questionnaire in collaboration individually recalled an average of $37.11(S D=9.41)$ information units, of which $3.99(S D=2.29)$, were distortions. Participants in the individual condition recalled an average of $35.41(S D=10.94)$ information units, of which $3.91(S D=$ 2.41) were distortions. The proportion of correct information recalled (from the video) between the two conditions was not significantly different, $t(150)=-1.06, p=.29(M=.25 ; S D=$ $.07 ; M=.24 ; S D=.08)$, neither were the distortions, $t(150)=$ $-.207, p=.84$.

To further analyze whether collaboration during the questionnaire influenced the final individual recall, we conducted a 2 (Recall task: R1 vs. R2; within-participants) $\times 2$ (Condition: Individual vs. Collaborative; between-participants) mixed ANOVA. The main effect of condition was not significant, $F(1,150)=.034, p=.85$. The main effect of recall task was significant, $F(1,150)=38.67, p<.001, \eta p^{2}=.205,90 \% \mathrm{CI}$ $[.12, .29]$, indicating that R2 was higher $(M=.25, S D=.07)$ than $\mathrm{R} 1(M=.23, S D=.07)$. The interaction between recall task and condition was also significant, $F(1,150)=18.36, p<$ $.001, \eta p^{2}=.109,90 \%$ CI $[.04, .19]$. Planned comparisons revealed that participants who collaborated during the questionnaire recalled more information in $\mathrm{R} 2(M=.25, S D=.07)$ than in $\mathrm{R} 1(M=.23, S D=.06), F(1,150)=55.16, p<.001, \eta p^{2}$ $=.269,90 \% \mathrm{CI}[.17, .36]$. For those who answered the questionnaire individually the difference between the two recall tasks was not significant, $\left(M_{R 2}=.24, S D=.08 ; M_{R 1}=.23\right.$, $S D=.08), F(1,150)=1.87, p=.174$.

\section{Recall of the information presented in the questionnaire}

Finally, to further examine whether the benefits of collaborating during the questionnaire generalized to a subsequent individual recall task we conducted a 2 (Condition: Individual vs. Collaborative; between-participants) $\times 2$ (Type of recalled information: Correct vs. Misinformation; within-participants) mixed ANOVA. Once again, a significant main effect of type of information, $F(1,150)=331.45, p<.001, \eta p^{2}=.688,90 \%$ CI $[.62, .74]$, indicated a higher recall of correct information
$(M=.23, S D=.13)$ than misinformation $(M=.04, S D=.06)$. The main effect of condition was not significant, $F(1,150)=$ $.551, p=.46$, that is, participants in both conditions recalled a similar amount of information from the questionnaire. The interaction between type of information and condition was significant, $F(1,150)=9.55, p=.002, \eta p^{2}=.060,90 \% \mathrm{CI}$ $[.01, .13]$ (see Fig. 2).

Planned comparisons indicated that both the participants who answered the questionnaire individually, $F(1,150)=$ $114.23, p<.001, \eta p^{2}=.432,90 \%$ CI $[.33, .51]$, and those who answered in collaboration, $F(1,150)=226.77, p<.001$, $\eta p^{2}=.602,90 \%$ CI $[.52, .66]$, recalled more correct information $\left(M_{\text {Ind }}=.21, S D=.13 ; M_{\text {CollQuest }}=.25, S D=.12\right)$ introduced by the questionnaire than misinformation $\left(M_{\text {Ind }}=.06\right.$, $\left.S D=.07 ; M_{\text {CollQuest }}=.03, S D=.04\right)$.

Importantly, those who answered the questionnaire in collaboration recalled significantly more correct information, $F(1,150)=3.95, p=.049, \eta p^{2}=.026,90 \%$ CI $[.00, .08]$, and less misinformation introduced by the questionnaire, $F(1,150)=5.11, p=.025, \eta p^{2}=.033,90 \%$ CI $[.00, .09]$, than those who answered individually.

Because the control group in both experiments was composed by the same participants, which introduces dependence between experiments, we conducted additional analyses comparing the three manipulations of the previous experiments: control (individual in all phases), collaborative in all phases, and collaborative only during questionnaire.

\section{Exploratory analysis}

In order to make the comparison between the recall conditions feasible, the individual recall performance was computed as in

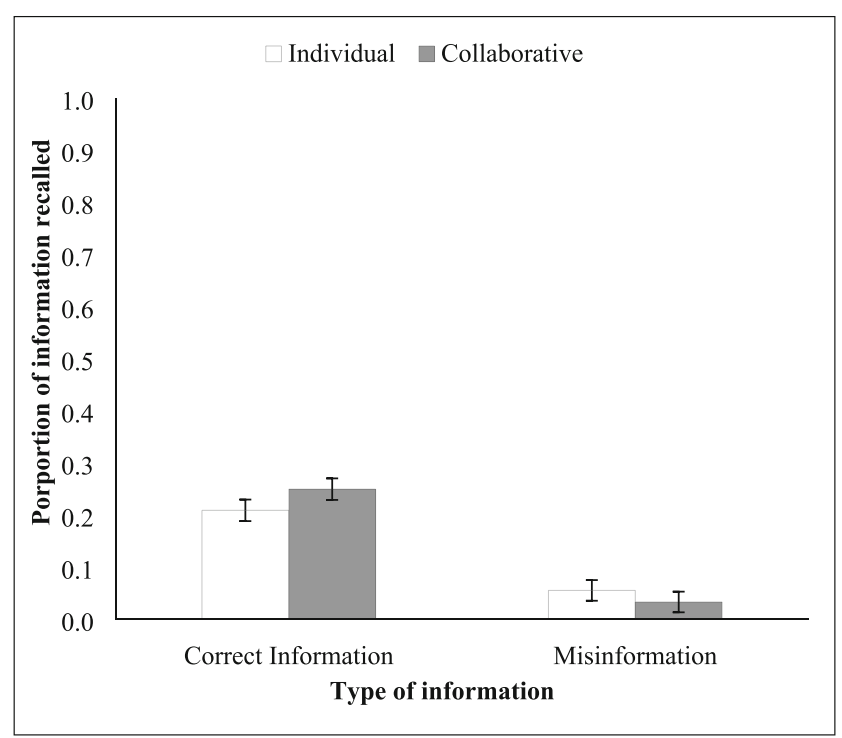

Fig. 2 Proportion of correct information and misinformation recalled from the questionnaire in Recall 2 by condition (error bars indicate $95 \%$ confidence interval) 
nominal groups to maintain the group as the statistical unit of analysis across conditions. The performance during the questionnaire was analyzed as in Experiments 1 and 2.

\section{Recall 1}

Two one-way ANOVA's revealed that the amount of distortions was not significantly different between the three conditions, $F(2,111)=1.08, p=.344$, but the proportion of correct information recalled was, $F(2,111)=31.37, p<.001, \eta p^{2}=$ $.361,90 \%$ CI $[.24, .45]$. Planned comparisons showed that correct recall $(M=.22, S D=.08)$ was significantly lower for those who collaborated than for those who worked individually $(M=.36, S D=.09 ;(M=.33, S D=.07), F(1,111)=54.70$, $p<.001 ; \eta p^{2}=.330,90 \%$ CI $[.21, .43]$ and $F(1,11)=37.85, p$ $<.001 \eta p^{2}=.254,90 \%$ CI $[.14, .36]$. These results replicated the collaborative inhibition effect. The difference between the two nominal groups was not significant, $F(1,111)=1.55, p=$ .216 .

\section{Correct information and misinformation accepted during the questionnaire}

A one-way ANOVA revealed significant differences between conditions, $F(2,149)=11.78, p<.001, \eta p^{2}=.137,90 \% \mathrm{CI}$ $[.06, .22]$, in the proportion of correct responses to the questionnaire. Planned comparisons showed that in the two conditions where participants collaborated during the questionnaire, the proportion of correct responses was higher than when participants responded individually, $F(1,149)=14.62, p<$ $.001, \eta p^{2}=.100,90 \%$ CI $[.04, .18]$ and $F(1,149)=16.76, p$ $<.001, \eta p^{2}=.101,90 \%$ CI $[.04, .18]$, respectively. No significant differences were observed between the two collaborative conditions, $F(1,149)=.055, p=.815$.

A mixed ANOVA 3 (Condition: Individual vs. Collaborative in all phases vs. Collaborative during the questionnaire) $\times 2$ (Type of accepted information: Misinformation vs. Correct) revealed that the main effect of condition was not significant, $F(2,149)=.245, p=.78$. The main effect of type of information was significant, $F(2,149)=1141.98, p<.001$, $\eta p^{2}=939,90 \%$ CI $[.92, .95]$, showing that correct information was more accepted $(M=13.10, S D=1.69)$ than misinformation $(M=6.36, S D=2.12)$. The interaction between condition and type of information was also significant, $F(2,149)=$ $11.78, p<.001, \eta p^{2}=137,90 \%$ CI $[.06, .22]$. As shown in the results of the planned comparisons presented in Experiments 1 and 2, in all three conditions, participants accepted more correct information than misinformation during the questionnaire.

Additionally, participants who responded individually to the questionnaire accepted less correct information than those who collaborated $F(1,149)=11.11, p=.001, \eta p^{2}=.069,90 \%$ CI $[.02, .14]$, and $F(1,149)=13.42, p<.001, \eta p^{2}=.083,90 \%$
CI $[.03, .16]$, respectively. The difference between the two collaborative conditions was not significant, $F(1,149)=$ $.081, p=.776$.

Finally, and as reported in the previous experiments, planned comparisons also revealed that participants who collaborated during the questionnaire accepted less misinformation than those who worked individually, $F(1,149)=3.85, p=$ $.05, \eta p^{2}=.025,90 \%$ CI $[.00, .08]$ and $F(1,149)=3.60, p=$ $.06, \eta p^{2}=.024,90 \%$ CI $[.00, .08]$. The difference between the two collaborative conditions was not significant, $F(1,49)=$ $.003, p=.956$.

\section{Recall 2}

The amount of distortions between the three conditions was not significantly different, $F(2,111)=.341, p=.712$, but the proportion of correct information recalled (from the video), was, $F(1,111)=40.94, p<.001, \eta p^{2}=.424,90 \%$ CI $[.30$, .51]. Planned comparisons showed that the recall of those who collaborated during R2 $(M=.22, S D=.08)$ was significantly lower than the recall of both nominal groups $(M=.36, S D=$ $.08 ; M=.37 ; S D=.08), F(1,111)=56.89, p<.001, \eta p^{2}=$ $.339,90 \%$ CI $[.22, .44]$ and $F(1,111)=65.61, p<.001, \eta p^{2}=$ $.372,90 \% \mathrm{CI}[.25, .47]$, replicating the collaborative inhibition effect. Overall, the difference between the two nominal conditions was not significant, $F(1,111)=.311, p=.578$. To further analyze whether collaboration during the questionnaire influenced the final recall, we conducted a 2 (Recall task: R1 vs. R2; within-participants) $\times 3$ (Condition: Individual vs. Collaborative in all phases vs. Collaborative during the questionnaire; between-participants) mixed ANOVA. The main effect of condition was significant, $F(2,111)=36.97, p<$ $.001, \eta p^{2}=.400,90 \%$ CI $[.28, .49]$, revealing that those who performed the recall tasks in collaboration recalled less information $(M=.22, S D=.08)$ than those who recalled individually $(M=.36, S D=.09 ; M=.35, S D=.08), F(1,111)=$ $57.94, p<.001, \eta p^{2}=.343,90 \%$ CI $[.23, .44]$, and $F(1,111)$ $=52.84, p<.001, \eta p^{2}=.323,90 \%$ CI $[.21, .42]$. Overall, the difference between the two nominal conditions was not significant, $F(1,111)=.117, p=.733$.

The main effect of recall task was significant, $F(2,1111)=$ $18.30, p<.001, \eta p^{2}=.248,90 \% \mathrm{CI}[.13, .34]$, revealing that performance in R2 $(M=.32, S D=.10)$ was significantly higher than R1 $(M=.30, S D=.10)$. Finally, the interaction between recall task and condition was also significant, $F(2,111)=16.98, p<.001, \eta p^{2}=.234,90 \%$ CI $[.12, .33]$. Planned comparisons showed that the recall performance improvement from R1 to R2 was only observed for those who recalled individually after collaborating during the questionnaire, $F(1,111)=51.76, p<.001, \eta p^{2}=.318,90 \%$ CI $[.20$, $.42]$. This difference was not significant for those who always worked individually, $F(1,111)=.348, p=.556$, or collaboratively, $F(1,111)=.141, p=.708$. 


\section{Recall of the information presented in the questionnaire}

A 3 (Condition: Individual vs. Collaborative in all phases vs. Collaborative during the questionnaire; between-participants) $\times 2$ (Type of recalled information: Correct vs. Misinformation; within-participants) mixed ANOVA revealed a significant type of information main effect, $F(1,111)=389.93, p<.001, \eta p^{2}=.778,90 \%$ CI $[.72, .82]$, namely a higher recall of correct information $(M=.35, S D=$ $.17)$ than misinformation $(M=.08, S D=.08)$. The main effect of condition was also significant, $F(2,111)=9.23, p<.001$, $\eta p^{2}=.143,90 \%$ CI $[.05, .23]$. Planned comparisons revealed that those who collaborated in all phases (namely during R2) recalled less information from the questionnaire $(M=.16, S D$ $=.16)$ than those who performed R2 individually $(M=.24, S D$ $=.18), F(1,111)=12.40, p<.001, \eta p^{2}=.101,90 \%$ CI $[.03$, $.19]$ and $(M=.25, S D=.22), F(1,111)=15.16, p<.001, \eta p^{2}=$ $.120,90 \% \mathrm{CI}[.04, .22]$. The difference between the two individual recall conditions was not significant $(M=.24, S D=$ $.22), F(1,111)=.138, p=.710$.

Finally, the condition $\times$ type of information interaction was significant, $F(2,111)=12.53, p<.001, \eta p^{2}=.184,90 \% \mathrm{CI}$ $[.08, .28]$, indicating that across the three conditions, the recall of correct information from the questionnaire was higher $\left(M_{\text {Coll }}=.26, S D=.16 ; M_{\text {Ind }}=.37, S D=.15 ; M_{\text {CollQuest }}=\right.$ $.42, S D=.16)$ than the recall of misinformation $\left(M_{\mathrm{Coll}}=.06\right.$, $S D=.07 ; M_{\text {Ind }}=.10, S D=.09 ; M_{\text {CollQuest }}=.06, S D=.06$ ), $F(1,111)=64.50, p<.001, \eta p^{2}=.368,90 \%$ CI $[.25, .46]$, $F(1,111)=122.81, p<.001, \eta p^{2}=.525,90 \%$ CI $[.42, .60]$, and $F(1,111)=227.69, p<.001, \eta p^{2}=.672,90 \%$ CI $[.59$, .73], respectively. Planned comparisons also showed that those who collaborated during R2 recalled less correct information than those who recalled individually, $F(1,111)=$ $10.39, p=.002, \eta p^{2}=.086,90 \%$ CI $[.02, .18]$ and $F(1,111)$ $=23.10, p<.001, \eta p^{2}=.172,90 \%$ CI $[.08, .27]$. This result replicated the collaborative inhibition effect. The difference between the two individual conditions was not significant, $F(1,111)=2.50, p=.116$. Critically, planned comparisons indicated that participants in the two conditions requiring collaboration during the questionnaire recalled less misinformation than those who answered the questionnaire individually, $F(1,111)=5.88, p=.017, \eta p^{2}=.050,90 \%$ CI $[.00, .13]$, and $F(1,111)=5.39, p=.022, \eta p^{2}=.046,90 \%$ CI $[.00, .12]$. The difference between the two collaborative conditions was not significant, $F(1,111)=.011, p=.918$. Overall, the exploratory analysis replicates the pattern of results reported in the previous experiments, namely that those who collaborated during the questionnaire provided more correct responses, accepted more correct information, and rejected more misinformation than those who worked individually. Additionally, participants in the collaborative condition recalled less correct information from the video and from the questionnaire than those who worked individually, replicating the collaborative inhibition effect. Importantly, participants from both collaborative conditions during the questionnaire recalled (individually or collaboratively) less misinformation than participants who worked individually during the questionnaire. Taken together these findings confirmed the role of discussion during collaboration in the acceptance of misinformation and its subsequent recall.

\section{General discussion}

The emergence and propagation of misinformation have important individual and social consequences. However, while the effects of exposure to false information for individual memory are well established, their examination in the context of social interaction has shown mixed results.

The current studies were designed to analyze the role of collaboration in encoding and retrieving misinformation introduced after encoding the original event. In Experiment 1, the collaborative manipulation occurred during the recall tasks and during the questionnaire. The results revealed that when responding to a questionnaire introducing correct information and misinformation regarding a previously encoded event, collaborative groups provided more correct responses than participants responding to the questionnaire individually. Specifically, when responding to this questionnaire, collaborative groups were more effective in accepting correct information (i.e., to consider that a statement is true when it corresponds to previously presented information) and tended to accept less misinformation (i.e., to consider that a statement is false when it does not correspond to an information presented). The higher performance observed in collaborative groups is in line with results reported in previous studies (e.g., Harris et al., 2012; Rossi-Arnaud et al., 2020; Vredeveldt et al., 2016, 2017), suggesting that the opportunity for discussion offered in collaborative groups working with the free-for-all method enhances the rejection of misinformation.

Collaborative groups were also expected to recall less misinformation in a subsequent recall task. However, while this pattern was actually observed, collaborative groups also recalled less correct information possibly due to a collaborative inhibition effect (Weldon \& Bellinger, 1997). Indeed, across recall tasks, participants in collaborative groups recalled less information from the original event than those in nominal groups, further documenting the role of collaboration in disrupting individual retrieval strategies (Basden et al., 1997). Therefore, Experiment 1 was not clear in showing whether the lower recall of misinformation in collaborative groups was due to error-pruning processes or simply reflects collaborative inhibition. To clarify this potential confound, in Experiment 2 the collaborative manipulation occurred only during the questionnaire where the misinformation was 
introduced. Again, the results revealed that participants collaborating during the questionnaire performed better, namely they provided more correct responses, accepted more correct information, and tended to accept less misinformation than those who worked individually. In a subsequent individual recall task, participants who collaborated during the questionnaire recalled more correct information than those who answered the questionnaire individually. Critically, participants who collaborated during the questionnaire also recalled less misinformation than those who answered the questionnaire individually. These results suggest that collaboration during the encoding of misinformation benefits future recall accuracy.

In both experiments some of the misinformation introduced by the questionnaire was included in R2, but its proportion was lower when participants collaborated during the questionnaire. While in Experiment 1 the lower recall of misinformation in collaborative groups (as compared to nominal ones) could reflect a collaborative inhibition effect, in Experiment 2, with all participants recalling individually, the lower recall of misinformation seems to result from collaborating during the questionnaire.

In line with the source-monitoring framework (Johnson et al., 1993; Roediger et al., 2001), our results seem to suggest that across experiments and conditions, there is a failure in monitoring the source of misinformation that is attributed to the source of the original event. However, this monitoring failure seems to be reduced when participants have the opportunity to discuss misinformation in a collaborative group and reach a consensus about its veracity. As mentioned before, the free-for-all method used in our paradigm allows free discussion and consensus among group members that is likely to enhance error-pruning mechanisms and the adoption of a conservative retrieval criterion (see Maswood \& Rajaram, 2019, for a review). These processes seem to reduce the production of false memories.

Finally, in Experiment 2 the recall of correct information from the original event increased from R1 to R2, but only for those who collaborated during the questionnaire. Previous studies have already shown that collaborating in successive recall tasks benefits subsequent individual recall (Blumen \& Rajaram, 2008). This is arguably the case because while each member of the group recalls information, the other members are re-exposed to the previously presented items. This re-exposure, which does not occur in individual conditions, can help memory consolidation (e.g., Blumen \& Rajaram, 2008; Blumen, Rajaram, \& Henkel, 2013). Experiment 2 further showed that such benefits of re-exposure can also be observed when collaboration occurs during encoding. To the best of our knowledge, this was the first study documenting the advantage of collaborative encoding in integrating correct information and rejecting misinformation in a subsequent individual recall task.
The results of the exploratory analyses further confirmed those findings. While participants in the collaborative condition recalled less information from the video and from the questionnaire than those who worked individually (i.e., nominal groups) replicating the collaborative inhibition effect, they also provided more correct responses, accepted more correct information, and rejected more misinformation during the questionnaire. Critically, participants from both collaborative conditions during the questionnaire recalled less misinformation than those who worked individually in all phases.

The procedure used in our study differs from the standard misinformation paradigm in that it offers participants in the collaborative conditions the opportunity to discuss misinformation at encoding. The observed results suggest that this discussion was critical in preventing misinformation in subsequent recall.

Additionally, the same number of correct and false statements was introduced to ensure that participants had the opportunity to discuss equal amounts of correct information and misinformation. This amount of misinformation provided could have led participants to become hypervigilant. According to the Discrepancy Detection principle (Tousignant, Hall, \& Loftus, 1986), misinformation is more likely to alter memory if the individual does not detect discrepancies between the original event and the misleading post event. Therefore, memory is more prone to errors if participants do not immediately detect this discrepancy. However, our results show that participants not only accepted and recalled misinformation but, importantly, the acceptance and recall of misinformation was moderated by the individual or collaborative nature of the tasks. Nevertheless, future studies should replicate these experiments, using different amounts of correct and misinformation.

Although our designs do not directly address the specific mechanisms operating during collaboration, the findings are clear in showing that collaboration during encoding can potentially reduce the acceptance of misinformation and that the benefits of this collaboration persist in a subsequent individual recall task.

In a time when information is circulating at a faster pace and in greater amounts than ever before, misinformation can increasingly be perceived as true and accurate, reshaping both personal and collective memory and, consequently, decisions and behaviors in relevant domains such as health, environment, or politics. Thus, it is of the highest scientific and societal importance to further uncover the socio-cognitive mechanisms shaping the acceptance and transmission of misinformation in social interaction contexts (Maswood \& Rajaram, 2019). This study represents a novel approach to the study of misinformation acceptance/rejection and transmission in the context of social interaction where misinformation often spreads. Specifically, this study further confirms that the 
opportunity for discussion during misinformation presentation has the potential to minimize its subsequent recall.

The production and dissemination of scientific knowledge emphasizing the role of discussion in preventing the acceptance and retrieval of misinformation is crucial to increase public awareness, as well as to inform public policies designed to mitigate its social transmission and ultimately to prevent people from engaging in behavior based on misinformation.

Funding Information These studies were supported by a grant awarded by the Portuguese Foundation for Science and Technology to the first and second authors (PTDC/MHC-PCN/5217/2014) and by funds awarded by the same foundation to the host institution of the third author (Psychology Research Centre - UID/PSI/01662/2019, School of Psychology, University of Minho).

\section{References}

Basden, B. H., Basden, D. R., Bryner, S., \& Thomas III, R. L. (1997). A comparison of group and individual remembering: Does collaboration disrupt retrieval strategies? Journal of Experimental Psychology: Learning, Memory, and Cognition, 23(5), 11761191. https://doi.org/10.1037/0278-7393.23.5.1176

Basden, B. H., Basden, D. R., Thomas III, R. L., \& Souphasith, S. (1998). Memory distortion in group recall. Current Psychology: Developmental, Learning, Personality, Social, 16(3-4), 225-246. https://doi.org/10.1007/s12144-997-1001-4

Blumen, H. M., \& Rajaram, S. (2008). Influence of re-exposure and retrieval disruption during group collaboration on later individual recall. Memory, 16(3), 231-244. https://doi.org/10.1080/ 09658210701804495.

Blumen, H. M., Rajaram, S., \& Henkel, L. (2013). The applied value of collaborative memory research in aging: Behavioral and neural considerations. Journal of Applied Research in Memory and Cognition, 2(2), 107-117. https://doi.org/10.1016/j.jarmac.2013.03.003

Deese, J. (1959). Influence of inter-item associative strength upon immediate free recall. Psychological Reports, 5(3), 305-312. https://doi. org/10.2466/pr0.1959.5.3.305

Gabbert, F., Memon, A., \& Allan, K. (2003). Memory conformity: Can eyewitnesses influence each other's memories for an event? Applied Cognitive Psychology, 17(5), 533-543. https://doi.org/10.1002/acp. 885.

Gallo, D. (2006). Associative illusions of memory: False memory research in DRM and related tasks. New York: Psychology Press.

Garcia-Marques, L., Garrido, M., Hamilton, D. L., \& Ferreira, M. B. (2012). Effects of correspondence between encoding and retrieval organization in social memory. Journal of Experimental Social Psychology, 48(1), 200-206. https://doi.org/10.1016/j.jesp.2011. 06.017

Garrido, M., Garcia-Marques, L., \& Hamilton, D. L. (2012a). Enhancing the comparability between part-list cueing and collaborative recall $\mathrm{A}$ gradual part-list cueing paradigm. Experimental Psychology, 59(4), 199-205. https://doi.org/10.1027/1618-3169/a000144

Garrido, M., Garcia-Marques, L., \& Hamilton, D. L. (2012b). Hard to recall but easy to judge: Retrieval strategies in social information processing. Social Cognition, 30(1), 56-70. https://doi.org/10.1521/ soco.2012.30.1.56

Harris, C. B., Barnier, A. J., \& Sutton, J. (2012). Consensus collaboration enhances group and individual recall accuracy. The Quarterly Journal of Experimental Psychology, 65(1), 179-194. https://doi. org/10.1080/17470218.2011.608590
Herrington, R. (Director) (2002). The stickup. EUA: Universal Pictures Video.

Johnson, M. K., Hashtroudi, S., \& Lindsay, D. S. (1993). Source monitoring. Psychological Bulletin, 114(1), 3-28. https://doi.org/10. 1037/0033-2909.114.1.3

Karns-Wright, T. E., Irvin, S. J., Suranic, S. L., \& Rivardo, M. G. (2009). Collaborative recall reduces the effect of a misleading post event. North American Journal of Psychology, 11(1), 17-28.

Loftus, E. F. (1975). Leading questions and the eyewitness report. Cognitive Psychology, 7(4), 560-572. https://doi.org/10.1016/ 0010-0285(75)90023-7

Loftus, E. F. (2005). Planting misinformation in the human mind: A 30 year investigation of the malleability of memory. Learning \& Memory, 12(4), 361-366. https://doi.org/10.1101/lm.94705

Loftus, E. F., \& Palmer, J. C. (1974). Reconstruction of automobile destruction: An example of the interaction between language and memory. Journal of Verbal Learning and Verbal Behavior, 13(5), 585-589. https://doi.org/10.1016/S0022-5371(74)80011-3

Luna, K., \& Migueles, M. (2008). Typicality and misinformation: Two sources of distortion. Psicológica, 29(2), 171-187.

Luna, K., \& Migueles, M. (2009). Acceptance and confidence of central and peripheral misinformation. The Spanish Journal of Psychology, 12(2), 405-413. https://doi.org/10.1017/s1138741600001797

Maki, R. H., Weigold, A., \& Arellano, A. (2008). False memory for associated word lists in individuals and collaborating groups. Memory \& Cognition, 36(3), 598-603. https://doi.org/10.3758/ MC.36.3.598

Marion, S. B., \& Thorley, C. (2016). A meta-analytic review of collaborative inhibition and postcollaborative memory: Testing the predictions of the retrieval strategy disruption hypothesis. Psychological Bulletin, 142(11), 1141-1164. https://doi.org/10.1037/bul0000071.

Maswood, R., \& Rajaram, S. (2019). Social transmission of false memory in small groups and large networks. Topics in Cognitive Science, 11(4), 687-709. https://doi.org/10.1111/tops.12348

McNabb, J. C., \& Meade, M. L. (2014). Correcting socially introduced false memories: The effect of restudy. Journal of Applied Research in Memory and Cognition, 3(4), 287-292. https://doi.org/10.1016/j. jarmac.2014.05.007

Meade, M. L., Nokes, T. J., \& Morrow, D. G. (2009). Expertise promotes facilitation on a collaborative memory task. Memory, 17(1), 39-48. https://doi.org/10.1080/09658210802524240

Meade, M. L., \& Roediger, H. L. (2002). Explorations in the social contagion of memory. Memory \& Cognition, 30(7), 995-1009. https://doi.org/10.3758/BF03194318.

Oliveira, H., Albuquerque, P. B., \& Saraiva, M. (2018). The study of false memories: Historical reflection. Trends in Psychology, 26(4), 17751785. https://doi.org/10.9788/TP2018.4-03En

Ost, J., Blank, H., Davies, J., Jones, G., Lambert, K., \& Salmon, K. (2013). False memory $\neq$ false memory: DRM errors are unrelated to the misinformation effect. PLOS ONE, 8(4):e57939. https://doi.org/ 10.1371/journal.pone.0057939

Otgaar, H., \& Candel, I. (2011). Children's false memories: Different false memory paradigms reveal different results. Psychology, Crime \& Law, 17(6), 513-528. https://doi.org/10.1080/ 10683160903373392

Otgaar, H., Candel, I., Scoboria, A., \& Merckelbach, H. (2010). Script knowledge enhances the development of children's false memories. Acta Psychologica, 133(1), 57-63. https://doi.org/10.1016/j.actpsy. 2009.09.002

Paulo, R. M., Albuquerque, P. B., Saraiva, M., \& Bull, R. (2015). The enhanced cognitive interview: Testing appropriateness perception, memory capacity and error estimate relation with report quality. Applied Cognitive Psychology, 29(4), 536-543. https://doi.org/10. 1002/acp.3132

Rajaram, S., \& Pereira-Pasarin, P. (2010). Collaborative memory: Cognitive research and theory. Perspectives on Psychological 
Science, 5(6), 649-663. https://doi.org/10.1177/ 1745691610388763

Rivardo, M. G., Rutledge, A. T., Chelecki, C., Stayer, B. E., Quarles, M., \& Kline, A. (2013). Collaborative recall of eyewitness event increases misinformation effect at 1 week. North American Journal of Psychology, 15(3), 495-512.

Roediger, H. L., \& McDermott, K. B. (1995). Creating false memories: Remembering words not presented in lists. Journal of Experimental Psychology: Learning, Memory, and Cognition, 21(4), 803-814. https://doi.org/10.1037/0278-7393.21.4.803

Roediger, H. L., Meade, M. L., \& Bergman, E. T. (2001). Social contagion of memory. Psychonomic Bulletin \& Review, 8, 365-371. https://doi.org/10.3758/BF03196174

Ross, M., Spencer, S. J., Blatz, C. W., \& Restorick, E. (2008). Collaboration reduces the frequency of false memories in older and younger adults. Psychology and Aging, 23(1), 85-92. https:// doi.org/10.1037/0882-7974.23.1.85

Rossi-Arnaud, C., Spataro, P., Bhatia, D., Doricchi, F., Mastroberardino, S., \& Cestari, V. (2020). Long-lasting positive effects of collaborative remembering on false assents to misleading questions. Acta Psychologica, 203, 102986. https://doi.org/10.1016/j.actpsy.2019. 102986

Saraiva, M., Albuquerque, P. B., \& Arantes, J. (2017). Production of false memories in collaborative memory tasks using the DRM paradigm. Psicologica, 38(2), 209-229.

Takahashi, M. (2007). Does collaborative remembering reduce false memories? British Journal of Psychology, 98(1), 1-13. https://doi. org/10.1348/000712606X101628.

Thorley, C., \& Dewhurst, S. A. (2007). Collaborative false recall in the DRM procedure: Effects of group size and group pressure.
European Journal of Cognitive Psychology, 19(6), 867-881. https://doi.org/10.1080/09541440600872068

Tousignant, J. P., Hall, D., \& Loftus, E. F. (1986). Discrepancy detection and vulnerability to misleading post-event information. Memory \& Cognition, 14(4), 329-338. https://doi.org/10.3758/BF03202511

Vredeveldt, A., Groen, R. N., Ampt, J. E., \& van Koppen, P. J. (2017). When discussion between eyewitnesses helps memory. Legal and Criminological Psychology, 22(2), 242-259. https://doi.org/10. 1111/lcrp.12097.

Vredeveldt, A., Hildebrandt, A., \& Van Koppen, P. J. (2016). Acknowledge, repeat, rephrase, elaborate: Witnesses can help each other remember more. Memory, 24, 669-682. doi:https://doi.org/10. 1080/09658211.2015.1042884

Weigold, A., Russell, E. J., \& Natera, S. N. (2014). Correction of false memory for associated word lists by collaborating groups. American Journal of Psychology, 127(2), 183-190. https://doi.org/10.5406/ amerjpsyc.127.2.0183

Weldon, M. S., \& Bellinger, K. D. (1997). Collective memory: collaborative and individual processes in remembering. Journal of Experimental Psychology. Learning, Memory, and Cognition, 23(5), 1160-1175. https://doi.org/10.1037/0278-7393.23.5.1160

Zhu, B., Chen, C., Loftus, E. F., Lin, C., \& Dong, Q. (2013). The relationship between DRM and misinformation false memories. Memory \& Cognition, 41(6), 832-838. https://doi.org/10.3758/ s13421-013-0300-2

Publisher's note Springer Nature remains neutral with regard to jurisdictional claims in published maps and institutional affiliations. 